\title{
RELAÇÕES ENTRE A EVAPOTRANSPIRAÇÃO DE UMA CULTURA DE BATATA (Solanum tuberosum, L.) E A EVAPORAÇÃO DO TANQUE CLASSE A. *
}

\author{
E. J. Scalopi ** \\ R. Scardua \\ A. E. KLAR **
}

\section{RESUMO}

\begin{abstract}
Neste trabalho, foram observadas as correlações e estabelecidos os coeficientes de proporcionalidade entre a evapotranspiração de uma cultura de batata, submetida a três regimes de umidade do solo, e a evaporação do tanque Classe $A$. Os dados obtidos mostram-se significativamente correlacionados, exceto quando o teor de umidade do solo era permitido atingir um valor correspondente a um potencial capilar de -2.0 bares, no estágio de formação e desenvolvimento dos tubérculos.

Para fins práticos, as relações obtidas entre a evapotranspiração e a evaporação do tanque Classe A foram simplificadas, de maneira que apenas três valores médios, representativos de três estágios característicos com relação à exigência em água, pođerão servir como critério para predizer as necessidades de irrigação da cultura, mesmo em perfodos relativamente curtos.
\end{abstract}

\section{INTRODUÇAO}

As informações a respeito da quantidade de água necessária para o desenvolvimento e produção das culturas, tem se constituido num problema frequentemente observado em regiões irrigadas, devido em grande parte, à dependência da evapotranspiração à inúmeros fatores naturais e humanos que geralmente variam com o local e o tempo (BLANEY, 1955).

Apesar do elevado número de métodos propostos para avaliar a evapotranspiração, nenhum parece desfrutar de uma preferência absoluta. De acordo com TANNER (1970), a escolha do método mais adequado varia com a precisão requerida no período de determinação, conveniência e custo.

As dificuldades encontradas na aplicação dos métodos hidrológicos e micrometereológicos (SCALOPI, 1972) proporcionaram o aparecimento de re-

- Entregue para publicacão em 25/9/73

* Departamento de Engenharia Rural - Fac. de Ciências Médicas e Biol. de Botucatu, SP

*** Departamento de Engenharia Rural - Escola Sup. de Agricultura \&Luiz de Queiroz», Piracicaba, S. P. 
lações empíricas para estimativa da evapotranspiração que, quando calibradas com os valores medidos através de qualquer método racional, podem fornecer dados razoavelmente quantitativos (TANNER, 1968).

Segundo FRITSCHEN e SHAW (1961), os dados obtidos através do uso de evaporímetros representam a integração do complexo de fatôres meteorológicos que, afetam a evapotranspiração. Admitem, porém, que existem diferenças de comportamento entre uma superfície de água livre e uma superfície natural vegetada, com relação às perdas de água. As principais diferenças foram atribuídas a:

a) variação nas características da superfície vegetada durante o ciclo de crescimento;

b) disponibilidade de água para o processo evaporativo e

c) absorção de energia pela superfície.

Os principais tipos de evaporímetros utilizados têm sido os tanques de evaporação e os atmômetros. Dentre os varios tipos existentes, o tanque Classe A, padronizado pelo United States Weather Bureau, tem merecido atenção especial (OLIVEIRA, 1971). TANNER (1968) admite que a localização dos atmômetros, geralmente em abrigos meteorológicos, resulta em complexas relações com a evapotranspiração. Além disso, a possibilidade de obstrução desfavorece o seu uso nestas estimativas.

FRITSCHEN e SHAW (1961) determinaram as relações entre a evapotranspiração e a evaporação do tanque Classe A para uma cultura de milho e recomendam o uso destas relações, ajustadas ao estágio de desenvolvimento da cultura, para predizer as necessidades de irrigação.

FUCHS e STANHILL (1963) encontraram relações lineares entre a evapotranspiração de uma cultura de algodoeiro e a evaporação de tanques Classe A, em uma grande variação de fatores climáticos, solos, plantas e condições de irrigação. Posteriormente, FUCHS e outros (1964) compararam a produção, aplicação de água e evapotranspiração de culturas de algodoeiro irrigadas de acordo com as relações evapotranspiração/evaporação anteriormente estabelecidas e, irrigadas de acordo com as determinações gravimétricas convencionais de umidade do solo. Encontraram resultados semelhantes, porém, acentuam que o método gravimétrico é muito mais trabalhoso.

DOSS e outros (1964) obtiveram uma elevada correlação entre a evapotranspiração de espécies forrageiras, submetidas a três regimes de umidade no solo e a evaporação de tanques Classe A. Aparentemente, as maiores correlações foram observadas nas espécies que cobrem totalmente a superfície do solo. Estabeleceram que as razões diárias da evapotranspiração podem ser estimadas para uma determinada cultura, estágio de crescimento e regime de umidade do solo, a partir de relações pré-estabelecidas com a evaporação do tanque.

SCARDUA (1970) encontrou coeficientes de proporcionalidade entre a evapotranspiração de uma cultura de milho e a evaporação do tanque Classe A, 
variaveis com o regime de umidade do solo e o estágio de desenvolvimento da cultura.

Neste trabalho, os autores pretenderam observar a correlação e determinar os fatores de conversão entre a evapotranspiração de uma cultura de batata (Solanum tuberosum, L.) submetida a três regimes de umidade do solo e a evaporação do tanque Classe $\mathrm{A}$, com a finalidade de serem utilizadas para estimativa das necessidades de água em projetos de irrigação.

\section{MATERIAL E MÉTODOS}

A parte experimental deste trabalho foi desenvolvida na Estação Experimental de Botucatu, Estado de São Paulo, localizada a 22052'55" de latitude sul e $48^{\circ} 26^{\prime} 22^{\prime \prime}$ de longitude oeste, a uma altitude média de $800 \mathrm{~m}$. O relêvo é normal e ondulado.

$O$ solo pertence a classe textural argila e apresenta um peso específico aparente médio de $1,5 \mathrm{~g} . \mathrm{cm}-{ }^{3}$ na camada de $0-60 \mathrm{~cm}$. O teor de umidade na capacidade de campo, determinada pelo método direto no campo, foi de $29,4 \%$ em relação ao peso do solo seco, médio para a profundidade $0-60 \mathrm{~cm}$. A curva característica de umidade do solo (Fig. 1) foi obtida procedendo-se conforme RICHARDS (1947, 1949).

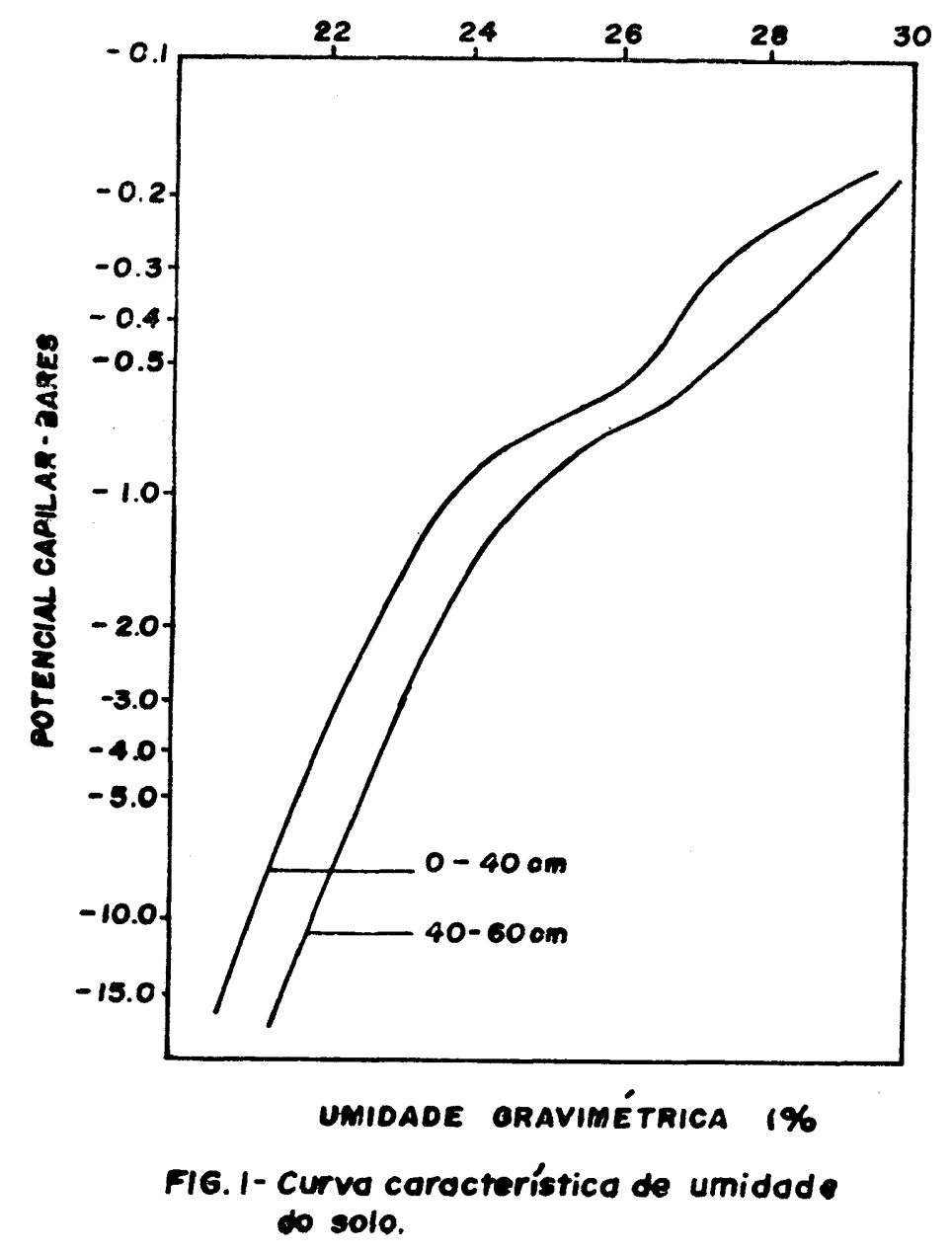


Para atender os objetivos propostos neste trabalho, instalou-se uma cultura de batata (Solanum tuberosum, L. cult. Aracy-IAC-2) a partir de tubérculos-sementes certificados, seguindo um esquema experimental de blocos casualizados, com três tratamentos e doze repetições. Cada parcela resumiu-se em três linhas de plantio, tendo $4 \mathrm{~m}$ de comprimento e espaçadas de $0,80 \mathrm{~m}$, das quais, considerou-se apenas a linha central como representativa dos tratamentos. Estes, foram definidos por regimes de umidade proporcionados à cultura, baseados na estimativa do potencial capilar de umidade do solo atingido antes de proceder a irrigação, através da determinação do teor de umidade gravimétrico e utilização da curva característica de umidade representada na Fig. 1. 0 quadro 1 caracteriza os referidos tratamentos.

Quadro 1 - Níveis mínimos médios de umidade do solo, em $40 \mathrm{~cm}$ de profundidade, nos tratamentos de irrigação.

\begin{tabular}{cccc} 
Tratamento & $\begin{array}{l}\text { Teor de umidade } \\
\text { \% de peso seco }\end{array}$ & $\begin{array}{l}\text { Potencial capi- } \\
\text { lar-bares }\end{array}$ & $\begin{array}{c}\text { \% de água dis- } \\
\text { ponível }\end{array}$ \\
\hline 3 & 27,0 & $-0,35$ & 75 \\
2 & 24,9 & $-0,80$ & 50 \\
1 & 22,8 & $-2,00$ & 25 \\
\hline
\end{tabular}

O plantio realizou-se em 24 de junho de 1971. O método de irrigação adotado foi sulcos em nível com os bordos fechados e a evapotranspiração foi determinada pelo método do balanço de água (SLATYER, 1967) a partir de determinações gravimétricas do teor de umidade do solo, cuja amostragem baseou-se nos resultados de ALLMARAS e GARDNER (1956).

Os dados evaporimétricos foram obtidos em um tanque Classe A construído segundo as normas fixadas pela Organização Mundial de Meteorologia (WMO) com chapa galvanizada AGW 20. As leituras de evaporação foram realizadas diariamente, utilizando-se um micrômetro de gancho, de fabricação Casella, tendo divisões de $0,01 \mathrm{~mm}$. O referido evaporímetro integra o Posto Meteorológico do Departamento de Física da Faculdade de Ciências Médicas e Biológicas de Botucatu, localizado a $700 \mathrm{~m}$ da área experimental.

A diferenciação entre os tratamentos foi iniciada 27 dias após o plantio, quando admitiu-se satisfatória a emergência das plantas no campo.

\section{RESULTADOS E DISCUSSÃO}

Os resultados obtidos estão graficamente representados na Fig. 2. Os dados relativos à evapotranspiração revelam variações na intensidade deste processo com o decorrer do desenvolvimento da cultura. 


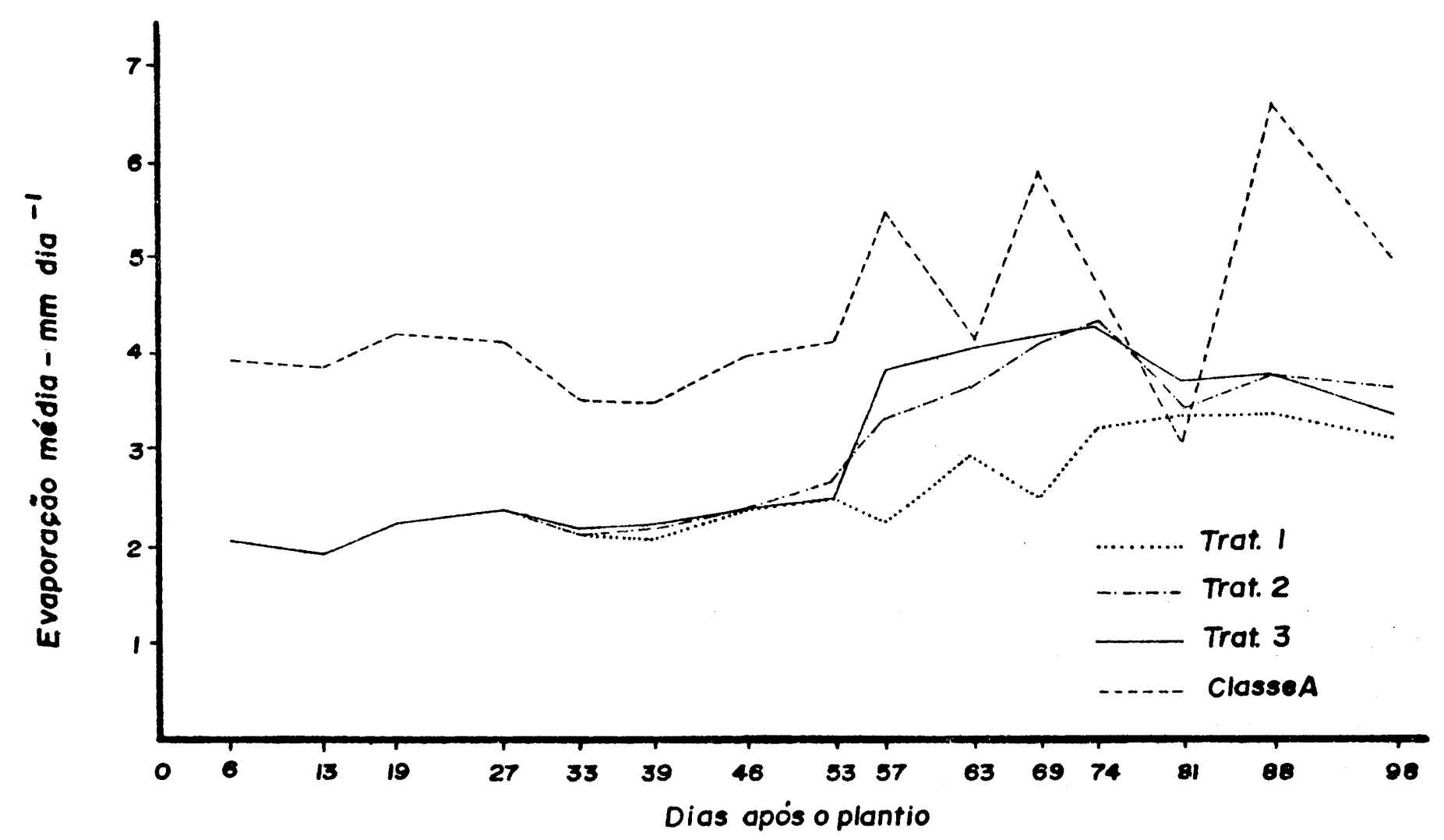

Fig.2 - Valores de evapotranspiraçäo determinados nos tres tratamentos e evaporacōo do tanque classe A em diferentes períodos do ciclo de crescimento da cultura.

Aparentemente, podem ser identificados três estágios bem definidos com relação à exigência em água pela cultura. $O$ primeiro, abrange o período inicial de crescimento, prolongando-se até aos 50 dias após o plantio, com uma razão de perda de água média de $2,2 \mathrm{~mm}$ por dia. Após este período, a razão de evapotranspiração aumentou sensívelmente, alcançando valores médios de 4,0 mm diários, como pode ser observado nos tratamentos onde o teor de umidade do solo não era reduzido a níveis inferiores àqueles correspondentes a um potencial capilar de $-0,80$ bar. Aos 80 dias, a evapotranspiração declinou, assumindo uma intensidade média diária de $3,6 \mathrm{~mm}$ nos tratamentos mais frequentemente irrigados ( 3 e 2) até aos 98 dias, quando as plantas apresentavam evidentes sintomas de maturação. Neste estágio, BOOCK (1963) recomenda suspender a irrigação e, consequentemente, não há maior interesse em se determinar a evapotranspiração.

No tratamento 1 , sujeito à ocorrência de níveis mais reduzidos de umidade no solo, a evapotranspiração foi limitada a $2,9 \mathrm{~mm}$ diários, no período caracterizado por uma maior exigência em água pela cultura. Conforme propõem de LIS e outros (1964), este período estaria associado à formação e desenvolvimento dos tubérculos.

As relações entre a evapotranspiração determinada nos três tratamentos e a evaporação do tanque Classe A estão representadas na Fig. 3. A variação dos valores assumidos por estas relações, durante o desenvolvimento da cultura, parecem seguir: um modelo semelhante aquele apresentado pelos dados de evapotranspiração, notadamente nos tratamentos com irrigações mais frequentes. Este fato permite considerar, para fins práticos, que ape- 


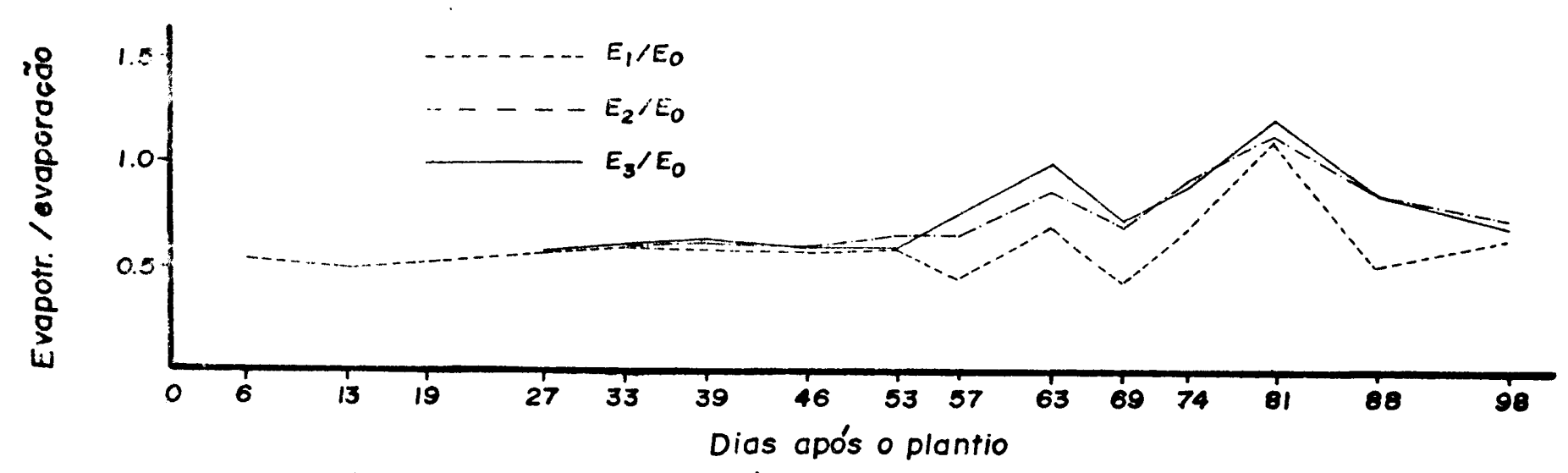

Fig. 3-Relações entre a evapotranspiraçõo e a evaporação do tanque ClasseA em diferentes períodos do ciclo dé crescimento da cultura.

nas três valores médios obtidos nestas relações poderiam representar os períodos caracterizados por um consumo de água semelhante. Entretanto, nos estudos de correlação, o estágio final do ciclo de crescimento foi incluido no 2. ${ }^{\circ}$ estágio considerado, devido ao número reduzido de dados disponíveis para efeito de cálculos (Quadro 2).

Quadro 2 - Valores de evapotranspiração (E) e evaporação do tanque Classe A (Eo) médios para três estágios de crescimento considerados e para o ciclo de crescimento da cultura.

Trat.

$\begin{array}{ccc}\text { Estágio } & \text { E média } & \text { Eo média } \\ \text { crescim. } & \mathrm{mm} . \mathrm{dia}^{-1} & \mathrm{~mm} \cdot \mathrm{dia}^{-1}\end{array}$

E / Eo $\quad r$

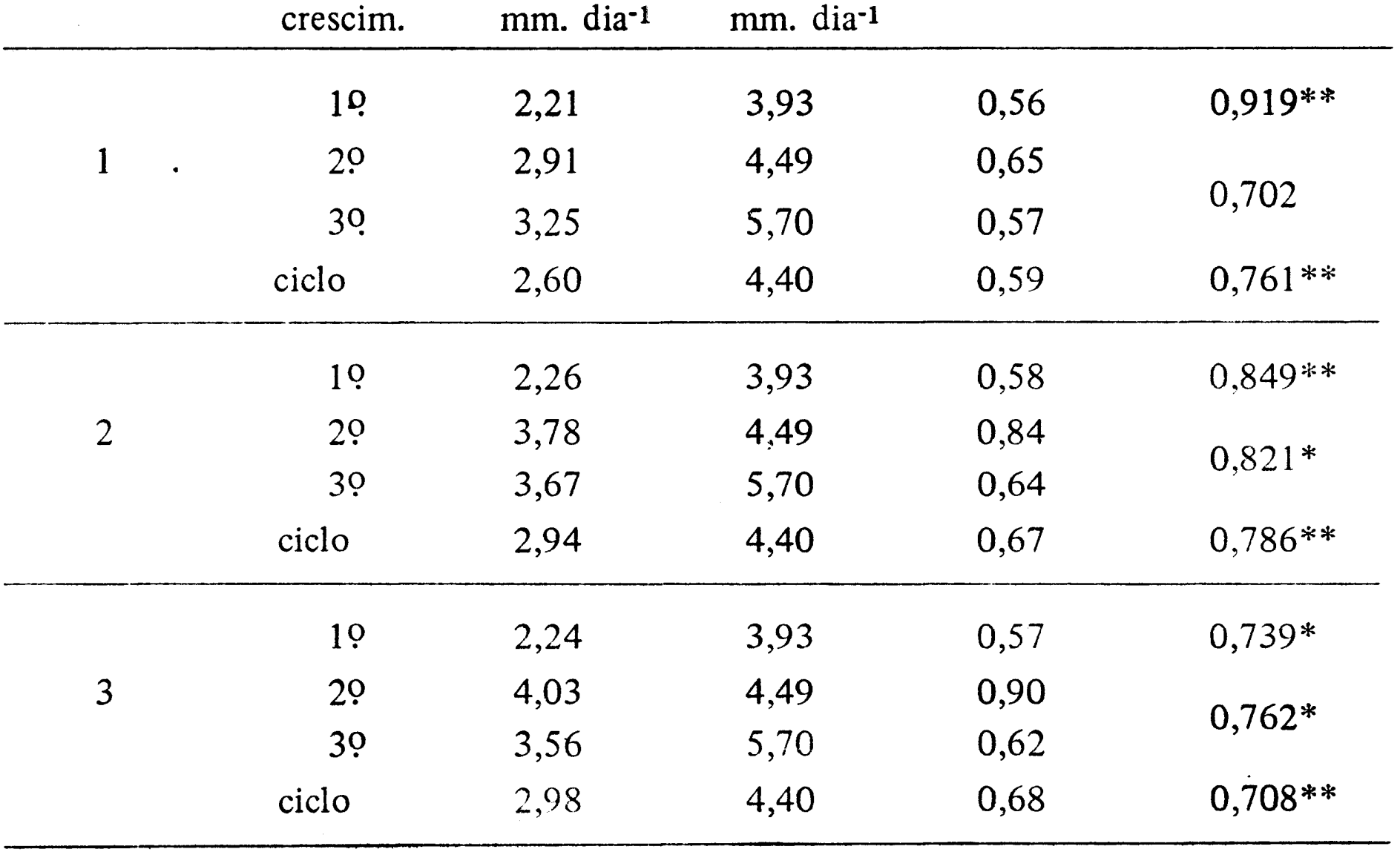


A evaporação do tanque Classe A foi significativamente correlacionada à evapotranspiração medida, exceto no segundo e terceiro estágios do tratamento 1. Este fato pode ser atribuído, provavelmente, à limitação da água do solo para as plantas, observada nos períodos caracterizados por um maior consumo o que provocou uma redução na intensidade de evapotranspiração.

A natureza deste método, em fornecer um valor integrado dos fatores meteorológicos envoividos no processo evaporativo, poderia explicar os elevados coeficientes de correlação observado em condições adequadas de umidade no solo (FRITSCHEN e SHAW, 1961).

SCARDUA (1970) encontrou para dois estágios de crescimento de uma cultura de milho, que os valores médios dos coeficientes de proporcionalidade entre evapotranspiração real e a evaporação de tanque Classe A, variaram de 0,42 a 0,69 nas parcelas não irrigadas, e 0,65 a 0,94 naquelas com maior frequência de irrigação. Seus resultados, médios para o ciclo de crescimento, obtidos nos tratamentos com níveis mínimos de 50 e $60 \%$ de água disponível no solo, coincidem com aqueles aqui apresentados, respectivamente nos tratamentos com níveis mínimos de 50 e $75 \%$. Além disso, os valores obtidos em cada estágio, individualmente, tiveram uma variação semelhante àqueles aqui apresentados.

Os trabalhos de DOSS e outros (1964), FUCHS e outros (1964) e TOVEY e outros (1969) parecem demonstrar que este método pode fornecer estimativas adequadas da evapotranspiração em períodos relativamente curtos. Por outro lado, os resultados mais satisfatórios parecem estar relacionados a condições de umidade do solo não limitantes e culturas que cobrem totalmente a superfície.

\section{CONCLUSÓES}

O uso de evaporímetros para predizer as necessidades de irrigação, como o tanque Classe $\mathrm{A}$, parece se constituir num método prático e econômico, além de fornecer estimativas adequadas, mesmo em períodos de alguns dias. Não havendo limitação de água do solo para a cultura, o método deverá tornar-se mais preciso, devido à semelhança de comportamento entre uma superfície vegetada e uma superfície livre de água, em relação ao processo evaporativo. No presente trabalho, as relações entre a evapotranspiração de uma cultura de batata e a evaporação do tanque Classe A foram simplificadas, de maneira que apenas três relações poderiam servir como referência para a estimativa da evapotranspiração; a primeira está relacionada ao período inicial de crescimento; a segunda, ao estágio de maior desenvolvimento vegetativo associado à formação e desenvolvimento dos tubérculos; e a terceira, representa o período final da fase vegetativa que precede a maturação das plantas. 


\section{SUMMARY}

\section{RELATIONSHIPS BETWEN EVAPOTRANSPIRATION OF A POTATO CROP (SOLANUN TUBEROSUM, L.) AND CLASS A PAN EVAPORA- TION.}

In this work were studied the correlation and the proportionality coefficients between the mesured evapotraspiration and Class A pan evaporation data, in a potato field-crop under three soil water regimes. The values obtained were significantly correlated, except when the soil moisture content reached a value corresponding to a capillary potential of about -2.0 bars from the stage of tuber growth to maturity.

To practical purposes the evapotraspiration/Class A pan evaporation relationship were simplifield, so that only three average values, representatives of three stages of plant water requeriments, could be a useful tool to predict the irrigation requirements of the crop, even in relatively short periods.

\section{LITERATURA CITADA}

ALLMARAS, R. R. e GARDNER, C. O. Soil sampling for moisture determinations on irrigations experiments. Agron. J. 48(1) : 15-17. 1956.

BLANEY, H. F. - Climate as an index of irrigation needs. Yb. Agric. U. S. - Dep. Agric.: Water. 341-345. 1955

BOOCK, O. J. Instruções para a cultura da batatinha. Bol. téc. Inst. agron. Est. Campinas 128. 1963 68p

DOSS, B. D., BENNETT, O. L. e ASHLEY, D. A. Moisture use by forage species as related to pan evaporation and net radiation. Soil Sci. 98(5): 322-327. 1964

FRITSCHEN, L. J. e SHAW, R. H. - Evapotranspiration for corn as related to pan evaporation. Agron. J. 53(3): 149-150. 1961

FUCHS, M., HAUSENBERG, I. e STANHILL, G. A field test of the control of cotton irrigation practice from class A pan data. Israel J. agric. Res. 14(4) : 273239. 1964.

e STANHILL, G. - The use of class A evaporation pan data to estimate the irrigation water requirement of the cotton crop. Israel J. agric. Res. 13(2) : 63-78. 1963.

LIS, B. R. de, PONCE, I e TIZIO, R. - Studies on water requirements of horticultural crops. I . Influence of drought at different growth stages of potato on tuber's yield. Agron. J. 56(5) : 377-381. 1964

OLIVEIRA, A. S. - Estudos comparativos da evaporaçăo potencial estimada por tanques e pelo método de Pennman. Tese de Livre-Docência apresentada à E.S.A. "Luiz de Queiroz" da Univ. São Paulo, 1971. 91 p. mimeografada.

RICHAPDS, L. A. - Pressure-membrane apparatus, construction and use. - Agric. Eng. 28: 451-454, 460. 1947.

- Methods of measuring soil moisture tension. Soil Sci. 68: 95-112. 1949.

SCALOPI, E. J. - Métodos climatológicos para avaliar a evapotranspiração. Dissertação apresentada à E. S. A. "Luiz de Queiroz" da Univ. São Paulo. 1972. 80 p. mimeogr. 
SCARDUA, R. Evapotranspiração real da cultura do milho como base aos projetos de irrigação. Tese de doutoramento apresentada à E. S. A. "Luiz de Queiroz" da Univ. São Paulo. 1970. 160 p. mimeogr .

SLATYER, R .O. - Plant-water relationship. New York, Academic press, 1967. $366 \mathrm{p}$.

TANNER, C. B -- Measurement of evapotranspiration. Agronomy. 11 :534-574. 1967. Water - Evaporation of water from plants and soil In Kozlowski, T. T. ed. W. p. 73-106. 
